\title{
Ritmo de piscar em portadores de pterígio antes e após a exérese
}

\author{
Eyeblink rate before and after pterygium exeresis
}

\author{
RodrigoUeno Takahagi ${ }^{1}$ \\ Fernando Gonçalves ${ }^{2}$ \\ Ricardo Kazuito Yamamoto ${ }^{3}$ \\ Magda Massae Hata Viveiros ${ }^{4}$ \\ Silvana Artioli Schellini ${ }^{5}$ \\ Carlos RobertoPadovani ${ }^{6}$
}

\begin{tabular}{l} 
RESUMO \\
\hline Objetivo: Avaliaro ritmo de piscar de portadores de pterígio antes e depois \\
da exérese. Métodos: Foram avaliados os movimentos palpebrais de 41 \\
pacientes antes e 60 dias depois da remoção cirúrgica da lesão. Os \\
movimentos palpebrais foram capturados durante 1 minuto, usando fil- \\
madora Sony Digital 8 DCR - TRV110, sob iluminação artificial, com o \\
indivíduo em posição primária do olhar, tendo como ponto de fixação a \\
própria filmadora. As imagens obtidas foram processadas por computa- \\
dor, quantificando-se o total de movimentos de piscar, o número de piscar \\
completo e incompleto, e as respectivas durações. Os dados foram sub- \\
metidos à análise estatística. Resultados: Antes da cirurgia 36,36\% dos \\
pacientes queixavam-se de sensação de corpo estranho e após a cirurgia, \\
61,02\% estavam assintomáticos. A avaliação do ritmo de piscar revelou \\
que a freqüência do piscar incompleto aumentou no pós-operatório. A \\
duração do piscar não se alterou antes e após a cirurgia. Conclusão: A \\
exérese do pterígio leva à diminuição dos sintomas irritativos. Após a \\
remoção da lesão, houve discreto aumento da freqüência de piscar in- \\
completo. Háa possibilidade da presença do pterígio não estar relacionada \\
com alterações do filme lacrimal, considerando que a alteração do ritmo de \\
piscar foi discreta. No entanto, outros estudos deverão ser realizados para \\
afirmar ou contestar esta hipótese.
\end{tabular}

Descritores: Pterígio/cirurgia; Aparelho lacrimal; Piscadela; Síndromes do olho seco

\section{INTRODUCÃO}

O pterígio é uma lesão sobrelevada na superfície ocular, acarretando alterações na camada de mucina, no tempo de ruptura e instabilidade do filme lacrimal ${ }^{(1-5)}$. Assim, é freqüente encontrar portadores de pterígio com queixas compatíveis com "olho seco".

A avaliação do filme lacrimal sem que se altere a sua estabilidade é difícil de ser feita. Os próprios exames diagnósticos, como o teste de Schirmer ou o teste de ruptura do filme lacrimal (BUT), podem induzir alterações, uma vez que necessitam da instilação de colírios para sua realização.

Piscar é a denominação que se dá para os movimentos de abertura e fechamento palpebral. Possui várias funções, como impedir a dessecação, eliminar corpos estranhos, excluir estímulos visuais, distribuir e manter o filme lacrimal $^{(6)}$. O piscar é dito completo, quando a pálbebra se fecha totalmente, ocultando o bulbo ocular; por outro lado, o piscar é chamado incompleto quando esta oclusão ocorre parcialmente.

Alterações da superfície ocular alteram o filme lacrimal e o ritmo de $\operatorname{piscar}^{(7)}$. O ritmo de piscar também se altera em algumas doenças ocula- 
res ${ }^{(8)}$, de acordo com o horário do dia (ritmo aumentado à noite) $)^{(9)}$, com o sexo (homens tem maior ritmo que mulheres) ${ }^{(10)}$ e com o nível de atenção ${ }^{(11)}$.

O piscar pode ser involuntário (espontâneo) ou voluntário, podendo ser influenciado por inúmeras condições, como luminosidade, temperatura, velocidade das correntes de ar, doenças oculares e pelo nível de atenção ${ }^{(8,11)}$. O piscar involuntário pode ser subdividido em piscar espontâneo (que é o que se repete em intervalos mais ou mesmos constantes) e o piscar reflexo (que ocorre por estimulação visual, auditiva ou tátil) ${ }^{(6)}$.

O ritmo de piscar em indivíduos normais é, em média, de 2 a 50 piscadas por minuto ${ }^{(12)}$. A velocidade de fechamento palpebral é de $14 \mathrm{~cm} /$ segundo $^{(6)}$. Para avaliar movimentos tão rápidos, é necessário usar meios especiais, sendo possível estimar o tempo de abertura e fechamento palpebral usando o programa iMovie ${ }^{(13)}$.

O filme lacrimal deficiente pode estar relacionado com o desenvolvimento do pterígio, devido à formação de "dellen" e micro-erosões epiteliais, que seriam o estímulo inicial para a proliferação do tecido fibrovascular ${ }^{(14)}$. Porém, parece não haver correlação entre a presença do pterígio e alterações do filme lacrimal ${ }^{(15-16)}$.

Tendo em vista a suposição de que o filme lacrimal possa estar alterado nos portadores de pterígio e que o ritmo de piscar possa ser um sinal indireto de que existe de fato esta alteração, nos propusemos a realizar este estudo que teve por objetivo avaliar o ritmo de piscar em portadores de pterígio antes e após a exérese da lesão.

\section{MÉTODOS}

Foram prospectivamente avaliados 41 portadores de pterígio, na Faculdade de Medicina de Botucatu-UNESP, dos quais 17 eram do sexo masculino e 24 , do feminino, com idade entre 21 e 71 anos. Trinta e três eram portadores de pterígio primário e 8 , recidivados.

Foram registrados os sintomas dos pacientes e realizado exame biomicroscópico, classificando-se a lesão, de acordo com a extensão, em: 1) Grau I - restrito ao limbo; 2) Grau II entre limbo e borda pupilar; 3) Grau III - na borda pupilar; 4) Grau IV - acometimento do eixo visual ou além deste. O pterígio grau II apresentou-se em maior proporção $(56,09 \%)$, seguido dos graus III $(26,82 \%)$, IV $(14,66 \%)$ e I $(2,43 \%)$.

Imediatamente antes da realização do procedimento cirúrgico, os movimentos palpebrais foram capturados durante 1 minuto, usando filmadora Sony Digital 8 DCR - TRV110, sob iluminação artificial (lâmpada fluorescente de $60 \mathrm{~W}$, a partir do teto de ambiente fechado), com o indivíduo em posição primária do olhar e fixando a filmadora, sempre no mesmo ambiente, no qual não havia outra atividade.

Após 60 dias do procedimento cirúrgico foram avaliadas as queixas dos pacientes e foi repetida a tomada de imagens digitais, usando-se a mesma técnica.
Todas as imagens obtidas foram processadas pelo computador McIntosh 400, usando-se o programa iMovie, quantificando-se o total de movimentos de piscar, o número de piscar completo e incompleto e a duração do tempo de abertura e fechamento palpebral.

Os dados foram submetidos a avaliação estatística, usando teste não-paramétrico de Wilcoxon para dados pareados, adotando-se o nível de significância de $5 \%$.

\section{RESULTADOS}

Na avaliação pré-operatória a queixa de sensação de corpo estranho foi a que se apresentou com maior freqüência $(36,36 \%)$, seguida de ardor/queimação $(28,78 \%)$, prurido $(22,72 \%)$, hiperemia $(7,57 \%)$ e lacrimejamento $(4,54 \%)$. Após 60 dias da cirurgia, houve somente queixas de hiperemia $(36,58 \%)$ e lacrimejamento $(2,4 \%)$, permanecendo o restante dos pacientes, assintomáticos (Gráfico 1).

A frequiência de piscar total no momento pré-cirúrgico foi, em média, de $11,00 \pm 10,65$, aumentando para $15,00 \pm 10,00$ no pósoperatório, com diferença estatística significativa. O mesmo ocorreu com a freqüência de piscar incompleto, que se apresentava com média de 8,00 \pm 5,25 piscar, elevando-se para 13,00 $\pm 9,00$ após a exérese da lesão, com aumento estatisticamente significativo. Analisando-se o piscar completo, observou-se freqüência praticamente mantida, com médias de 2,00 $\pm 2,75$ e 2,00 \pm 2,50, antes e após a cirurgia, respectivamente (Tabela 1).

A duração do tempo de abertura e fechamento palpebral de piscar completo foi, em média, de 0,43 $\pm 0,25$ segundos previamente à cirurgia e $0,43 \pm 0,28$ segundos após a mesma, sem diferença estatística significativa. Também não houve diferença entre as médias da duração do tempo de abertura e fechamento palpebral de piscar incompleto que foram $0,45 \pm$ 0,06 segundos e 0,44 $\pm 0,05$ segundos nos momentos pré e pós-operatório, respectivamente (Tabela 1).

\section{DISCUSS ÃO}

A maioria dos portadores de pterígio apresentava sintomas de sensação de corpo estranho, prurido, ardor e lacrimejamento. Os sintomas mais pronunciados estão relacionados com a elevação da cabeça, secura e "dellen"(1), justamente nos pterígios maiores, como comprovado na população estudada.

Após a remoção do pterígio, houve aumento da freqüência de piscar, às custas do aumento do piscar incompleto. Este modo de piscar que está bastante presente em piscar espontâneo ${ }^{(6)}$, é importante na manutenção da umidade e lubrificação da superfície ocular ${ }^{(17)}$. Na realidade, se a presença do pterígio estivesse relacionada com "olho seco", com a remoção da lesão, o esperado seria que houvesse regularização da superfície ocular e menor ritmo de piscar.

Indivíduos submetidos à cirurgia refrativa apresentam instabilidade do filme lacrimal após o procedimento em decorrên- 


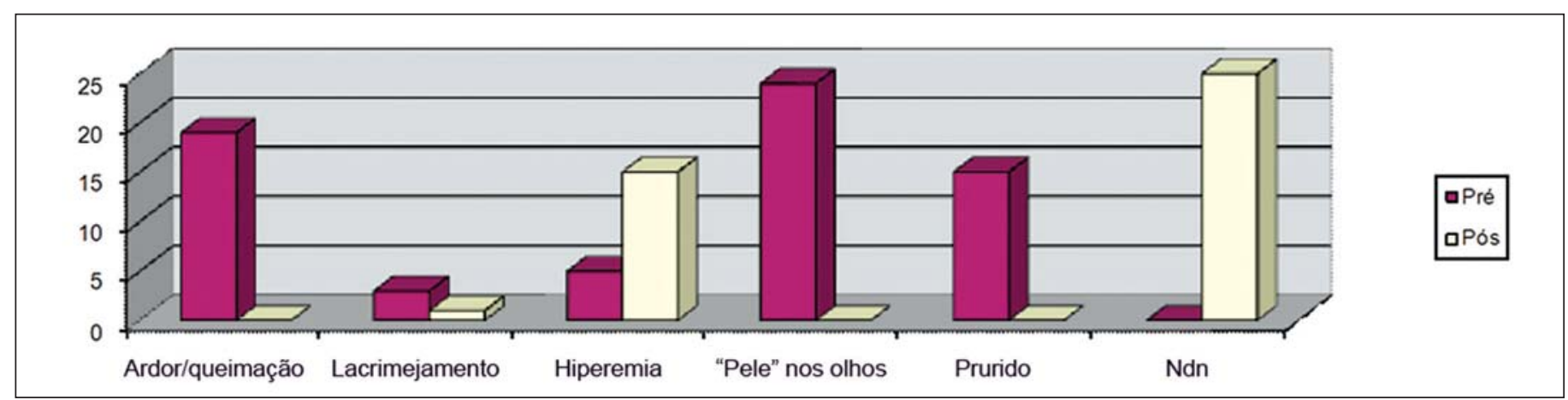

Gráfico 1 - Distribuição dos pacientes segundo queixas no período antes e 60 dias após a cirurgia

\begin{tabular}{|c|c|c|c|c|}
\hline \multirow[b]{2}{*}{ Variável } & \multirow[b]{2}{*}{ Piscar } & \multicolumn{2}{|c|}{ Momento } & \multirow[b]{2}{*}{ Resultado do teste } \\
\hline & & Antes & Depois & \\
\hline \multirow{3}{*}{ Freqüência } & Completo & $\begin{array}{c}2,00 \pm 2,75 \\
(0,00-51,00)\end{array}$ & $\begin{array}{l}2,00 \pm 2,50 \\
(0,00-52,00)\end{array}$ & $0,53(P<0,01)$ \\
\hline & Incompleto & $\begin{array}{c}8,00 \pm 5,25 \\
(0,00-45,00)\end{array}$ & $\begin{array}{l}13,00 \pm 9,00 \\
(0,00-41,00)\end{array}$ & $2,61 \quad(P<0,01)$ \\
\hline & Total & $\begin{array}{l}11,00 \pm 10,65 \\
(0,00-51,00)\end{array}$ & $\begin{array}{c}15,00 \pm 10,00 \\
(1,00-66,00)\end{array}$ & $2,51 \quad(P<0,05)$ \\
\hline \multirow{3}{*}{$\begin{array}{l}\text { Duração } \\
\text { do movimento }\end{array}$} & Completo & $\begin{array}{c}0,43 \pm 0,25 \\
(0,00-0,78)\end{array}$ & $\begin{array}{c}0,43 \pm 0,28 \\
(0,00-0,84)\end{array}$ & $0,67(P>0,05)$ \\
\hline & Incompleto & $\begin{array}{c}0,45 \pm 0,06 \\
(0,00-0,59)\end{array}$ & $\begin{array}{c}0,44 \pm 0,05 \\
(0,00-0,60)\end{array}$ & $1,64(P>0,05)$ \\
\hline & Total & $\begin{array}{c}0,81 \pm 0,22 \\
(0,00-1,10)\end{array}$ & $\begin{array}{c}0,85 \pm 0,27 \\
(0,00-1,26)\end{array}$ & $1,33(P>0,05)$ \\
\hline
\end{tabular}

cia de lesão corneana e diminuição da sensibilidade ${ }^{(18)}$. A remoção do pterígio resulta, de imediato, em alteração da superfície ocular que poderia interferir com o estímulo reflexo da glândula lacrimal, diminuindo a lubrificação e acarretando maior freqüência de piscar. Além disso, as irregularidades existentes logo após a exérese poderiam interferir na integridade da superfície ocular, prejudicando a lubrificação e contribuindo para o maior número de piscar.

Para evitar estes fatores que poderiam estar presentes na fase em que não existe cicatrização completa, optou-se por realizar a segunda avaliação 60 dias após o procedimento cirúrgico. Assim, observou-se aumento da freqüência de piscar incompleto. Porém, duração de piscar completo e incompleto, antes e após a exérese da lesão, não se alteraram.

Após a remoção do pterígio, houve alteração do ritmo de piscar às custas do aumento do piscar incompleto. A exérese do pterígio provoca erosão corneana e redução da quantidade de conjuntiva, sítio que contém estruturas produtoras da mucina do filme lacrimal. Estes fatores poderiam contribuir para uma provável instabilidade do filme lacrimal, com conseqüente aumento do piscar. Também após a cirurgia de LASIK, quando ocorre interferência com a inervação corneana, há alteração da dinâmica lacrimal na superfície ocular ${ }^{(18)}$.

\section{CONCLUSÃO}

A exérese do pterígio leva à diminuição dos sintomas irritativos. Após a remoção da lesão, houve discreto aumento da freqüência do piscar incompleto. Há a possibilidade da presença do pterígio não estar relacionada com alterações do filme lacrimal, considerando que a alteração do ritmo de piscar foi discreta. No entanto, outros estudos deverão ser realizados para afirmar ou contestar esta hipótese.

\section{ABSTRACT}

Purpose: To evaluate the eyeblink rate in patients with pterygium before and after the lesion exeresis. Methods: Forty-one patients with pterygium were evaluated before and 60 days after the lesion exeresis. They were digitally videotaped during one minute in a standard setting using a Sony Digital 8 DCR - TRV110 and the images were transferred to a personal computer (McIntosh 400) and processed with the iMovie software. The patients stayed in primary gaze position, under artificial ilumination. Total blink, complete and incomplete blink and the opening and closure time were measured using frames scale. The data were submitted to statistical analysis. 
Results: Before surgery, $36.36 \%$ of the patients with pterygium complained of foreign body and after that, $61.02 \%$ had no symptoms. The incomplete blink increased after surgery. The opening and closure time was similar before and after surgery. Conclusion: With pterygium exeresis the symptoms decreased and the incomplete blink increased a little. Nevertheless the blink rate barely changed. Further research needs to be provided to confirm this hypothesis.

Keywords: Pterygium/surgery; Lacrimal apparatus; Blinking; Dry eye syndromes

\section{REFERÊNCIAS}

1. Paton D. Pterygium management based upon a theory of pathogenesis. Trans Am Acad Ophthalmol Otolaryngol. 1975;79:603-5.

2. Marzeta M, Toczolowski J. [Study of mucin layer of tear film in patients with pterygium]. Klin Oczna.2003;105(1-2):60-2. Polish.

3. Ishioka M, Shimmura S, Yagi Y, Tsubota K. Pterygium and dry eye. Ophthalmologica. 2001;215(3):209-11.

4. Kadayifçilar SC, Orhan M, Irkeç M. Tear functions in patients with pterygium. Acta Ophthalmol Scand. 1998;76(2):176-9.

5. Rajiv MS, Mithal S, Sood AK. Pterygium and dry eye - a clinical correlation. Indian J Ophthalmol. 1991;39(1):15-6.

6. Recordes M. Physicology of the brown and eyelid. In: Tasman W, Jaeger EA, editors. Duane's clinical ophthalmology [CD-ROM]. Philadelphia: JB Lippincott; 1997

7. Tsubota K, Nakamori K. Effects of ocular surface area and blink rate on tear dynamics. Arch Ophthalmol. 1995;113(2):155-8.

8. Dumery B, Toi VV. Relationship between blink rate, ocular desconfort and visual tasks [abstract]. Invest Ophthalmol Vis Sci. 1997;38(Suppl):S68.

9. Barbato G, Ficca G, Muscettola G, Fichele M, Beatrice M, Rinaldi F. Diurnal variation in spontaneous eye blink rate. Psychiatry Res. 2000;93(2):145-51.

10. Bentivoglio AR, Bressman SB, Cassetta D, Carretta D, Tonali P, Albanese A. Analysis of blink rate patterns in normal subjects. Mov Disord. 1997;12(6):1028-34.

11. Sahlin S, Chen E. Gravity, blink rate and lacrimal drainage capacity. Am J Ophthalmol. 1997;124(6):758-64. Comment in: Am J Ophthalmol. 1998;126(1):152.

12. Monster AW, Chan HC, O'Connor D. Long trends in human eye blink rate. Biotelem Patient Monit. 1978;5(4):206-22.

13. Schellini SA, Sampaio AA Jr, Hoyama E, Cruz AA, Padovani CR. Spontaneous eye blink analysis in the normal individual. Orbit. 2005;24(4):239-42.

14. Barraquer MJ. [Localized discontinuity of the precorneal lacrimal film. Etiology of Fuchs' marginal corneal ulcers, of progression of pterygium and of certain corneal necroses in the neighborhood of keratoprostheses and keratoplasties.] Ophthalmologica. 1965;150(2):111-22. French.

15. Alves MR, Abreu LFM, Sampaio MW, Zanato A, Magalhães PB. Contribuição ao estudo da ação do filme lacrimal na evolução do pterígio. Rev Bras Oftalmol. 1985;44(3):94-5.

16. Bittencourt LCF, Kureski ML, Santos L, Arana J. Estudo do filme lacrimal em pacientes portadores de pterígio. Rev Bras Oftalmol. 1996;55(1):33-8.

17. Cruz AAV. Blefaroptoses e retrações palpebrais. São Paulo: Cultura Médica. 1998.

18. Battat L, Macri A, Dursun D, Pflugfelder SC. Effects of laser in situ keratomileusis on tear production, clearance, and the ocular surface. Ophthalmology. 2001;108(7):1230-35. Comment in: Ophthalmology. 2002;109(11):1947-8; author reply 1948; Ophthalmology. 2002;109(11): 1948-9; author reply 1949. 